\title{
Bedarf an neuen Tbc-Medikamenten
}

\author{
Tuberkulose ist wieder zum Thema geworden. Wichtig: Auch bei unspezifischen Symptomen \\ sollte man sie in die Überlegungen einbeziehen. Moderne Sequenzierungstechniken erlauben \\ heute eine rasche Diagnose und eine zeitnahe Resistenzbeurteilung.
}

\begin{abstract}
„Mit der Migration kommen nicht nur neue Menschen nach Deutschland, sondern auch Infektionserreger, die bisher nicht vorrangig differenzialdiagnostisch diskutiert wurden und auch teils in Vergessenheit geraten sind", so Prof. Thomas Welte, Hannover. Dazu gehöre vor allem die Tuberkulose ( $\bullet$ Abb. 1). Denn deren Prävalenz sei in Ländern, aus denen die Menschen fliehen, wesentlich höher als bei uns. Dies gilt besonders für Flüchtlinge aus Osteuropa und den Balkanstaaten, Afghanistan, Pakistan und dem westlichen Afrika, weniger für Menschen aus dem Irak oder Syrien. Anders als in Deutschland sind extrapulmonale Manifestationen der Tbc (Lymphknoten, Magen-Darm- und Urogenitaltrakt), aber auch septische Verläufe bei Migranten häufiger als bei Deutschen. Man müsse bedenken, dass Flüchtlinge deutlich geschwächt und immuninkompetent seien, was den Krankheitsverlauf ungünstig beeinflusse, so Welte.
\end{abstract}

\section{Schnelle und zuverlässige Resistenzbestimmung}

Neueste Entwicklungen in der Sequenziertechnologie, nämlich das Next Generation Sequencing (NGS), machen heute eine in wenigen Tagen vorliegende, sichere und kostengünstige Sequenzierung des gesamten Genoms der Mykobakterien möglich, sodass auf eine genetisch determinierte Suszeptibilität für die Medikamente bzw. eine Resistenz gegenüber antituberkulösen Substanzen (MDR/XDR-Tb) nachgewiesen werden kann. Die Sensitivität dieser Methode liegt bei über $90 \%$ und ist damit dem konventionellen Vorgehen mittels Kultur deutlich überlegen [1]. „Bei mikroskopisch positiven Sputen ist heute der Nachweis von resistenz- bzw. suszeptibilitätdefinierenden Mutationen und so eine komplette Sensibilitätstestung bzgl. sämtlicher Erst- und Zweitlinien-Medikamenten ohne vorherige Kultur möglich, so-

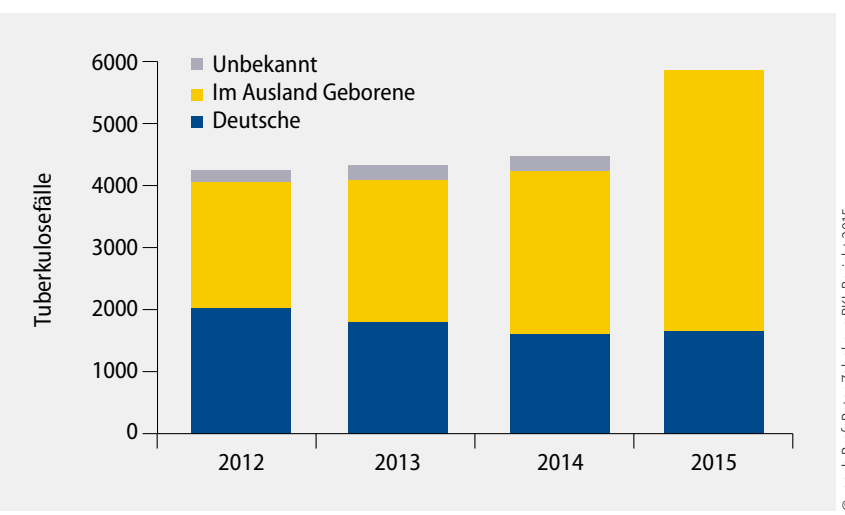

1 Die Zahl der Tuberkulosefälle in Deutschland steigt - vorrangig getrieben durch die Migration aus Ländern wie den Balkanstaaten, Afghanistan, Pakistan und des westlichen Afrika. dass das Verfahren in absehbarer Zeit in den Workflow der Tuberkulose-Diagnostik eingehen dürfte", so Prof. Peter Zabel, Ärztlicher Direktor der Medizinischen Klinik Borstel.

Von einer latenten $\mathrm{Tbc}$ spricht man, wenn der Interferon Gamma Release Assay (IGRA) positiv ist, ohne dass Hinweise für eine aktive Tbc vorliegen. Der Test wird eingesetzt als Screeningverfahren bei Kontaktpersonen zu Tbc-Erkrankten. Bei einem positiven Befund besteht allerdings nur ein sehr geringes Risiko für eine manifeste Erkrankung. „Eine präventive Chemotherapie reduziert zwar das Tuberkuloserisiko, aber die NNT liegt bei 38, die ist also relativ hoch", so Zabel [2].

\section{Dosiserhöhung bei Rifampicin nicht effektiv}

Seit über 40 Jahren wird Rifampicin in einer Dosierung von 10 $\mathrm{mg} / \mathrm{kg}$ KG und maximal 600 mg/Tag zur kombinierten Tbc-Behandlung empfohlen. Eine neue Studie ergab nun, dass eine Erhöhung der Dosis auf $15 \mathrm{mg} / \mathrm{kg}$ KG bei der tuberkulösen Meningitis nicht effektiv ist, allerdings könnte eine intravenöse Applikation vorteilhaft sein $[3,4]$. Eine adjuvante Gabe von Vitamin D ist auch nicht hilfreich bei der Therapie der pulmonalen Tbc. Offen aber bleibt, ob eine Vitamin-D-Gabe präventiv wirkt oder eine Reaktivierung verhindern kann.

Im letzten Jahr sind zwei neue Substanzen für die Tbc-Therapie zugelassen worden, nämlich Bedaquilin und Delamanid. Sie werden eingesetzt bei Patienten mit einer MDR/XDR-Tb und zwar zusätzlich, wenn aufgrund der Resistenzlage eine effektive Fünffach-Therapie mit den bisher verfügbaren Substanzen nicht möglich ist. „Diese Substanzen ermöglichen eine an die individuelle Resistenzlage angepasste personalisierte Therapie“, so Zabel. Die Studien zeigen in Kombination mit Pyrazinamid und Moxifloxacin eine hohe Effektivität und gute Verträglichkeit [5, 6]. Auch erwies sich die neue Kombination aus Moxifloxacin, Pretomanid und Pyrazinamid in Form einer achtwöchigen Initialtherapie bei sensibler und bei MDR-TB als effektiver als der bisherige Standard [7]. ,Doch vor einem breiten und unkontrollierten Einsatz dieser neuen Substanzen muss im Hinblick auf Resistenzbildung gewarnt werden“, so Zabel. Der Einsatz sollte auch wegen der Nebeneffekte und Interaktionen auf qualifizierte Zentren beschränkt sein. Dr. med. Peter Stiefelhagen

Literatur:

1. Walker TM et al.: Lancet Infect Dis. 2015 Oct; 15(10):1193-202

2. Zellweger JP et al.: A J Respir Crit Care Med. 2015 May 15;191(10):1176-84

3. Heemskerk et al.: N Engl J Med. 2016 Jan 14; 374(2):124-34

4. Crevel R et al.: N Engl J Med. June 2, 2016; 374; 22:2187-89

5. Olaru ID et al.: Ann Am Thorac Soc. 2016 Aug; 13(8):1271-8

6. Olaru ID et al.: J Antimicrob Chemother. 2016 Apr; 71(4): 852-4

7. Dawson R et al.: Lancet. 2015 May 2; 385(9979):1738-47

Quelle: PneumoUpdate 2016, 18. November 2016 in Wiesbaden 